Vardhini Padmanabhan*, Punitha Namadurai and Ramakrishnan Swaminathan

\title{
Analysis of Preterm Pregnancies using Empirical Mode Decomposition based Fractal Features
}

\begin{abstract}
:
Preterm birth (gestational age $<37$ weeks) is a serious pregnancy related complication that could lead to fetal morbidity and mortality. Monitoring the activity of uterus is considered to be crucial for the early diagnosis of preterm birth. Uterine Electromyography ( $\mathrm{uEMG}$ ) is a non-invasive technique that provides a quantitative measure of uterine activity from the abdominal surface. In this work, an attempt has been made to characterize preterm uEMG signals using Empirical Mode Decomposition based Detrended Fluctuation Analysis (EMD-DFA). Preterm signals with varied gestational ages are considered from an online database. EMD-DFA is applied on these signals to compute the fluctuation function. The double-logarithmic plot of fluctuation function versus scale is evaluated and Chi-square analysis is performed for identifying linear scaling regions. Five features namely shortterm exponent $(H s)$, long-term exponent $(H l)$, inflection point, short-term fractal angle $\left(\alpha_{H s}\right)$ and long-term fractal angle $\left(\alpha_{H l}\right)$ are extracted and analyzed. Further, Coefficient of Variation (CV) is computed to examine the variations of these features among different subjects. Results show that EMD-DFA is able to characterize the fluctuations of preterm signals. From the double-logarithmic plot, a slow variation of fluctuation function is observed with respect to scale when the time to delivery is more. This indicates the presence of rapid signal fluctuations in the early stages of pregnancy. Based on the feature values, it is observed that the signal fluctuations are more correlated and smoother as the time to delivery approaches. Among the extracted features, $\mathrm{CV}$ values of $H s$,
\end{abstract}

\footnotetext{
*Corresponding author: Vardhini Padmanabhan: Research Scholar, Department of Applied Mechanics, Indian Institute of Technology Madras, Chennai, Tamilnadu, India

e-mail: niidvardhini@gmail.com

Punitha Namadurai: Assistant Professor, Department of Biomedical Engineering, SSN College of Engineering, Chennai, Tamilnadu, India

Ramakrishnan Swaminathan: Professor, Department of Applied Mechanics, Indian Institute of Technology Madras, Chennai, Tamilnadu, India
}

$H l, \alpha_{H s}$ and $\alpha_{H l}$ are observed to be low indicating that these features have least inter-subject variations in preterm signals. The EMD-DFA based fractal features show the ability to detect the subtle variations in uEMG signals. As early diagnosis of preterm delivery is imperative for timely medical intervention and treatment, it appears that the proposed approach could aid in determining the changes in uterine contractions in preterm condition.

Keywords: Uterine Electromyography, Preterm pregnancies, Empirical mode decomposition based detrended fluctuation analysis, Fluctuation function.

https://doi.org/10.1515/cdbme-2021-2200

\section{Introduction}

Preterm birth is referred to as the birth of babies before 37 gestational weeks. It is a major cause for mortality and morbidity of newborns worldwide. Monitoring uterine activity is considered crucial for early preterm birth diagnosis [1].

Uterine Electromyography (uEMG) records the uterine electrical activity from the abdomen non-invasively. An objective measure is obtained as it examines the electrical potentials that underlie the uterine contractions. As a result, frequency, intensity and duration of the contractions could be evaluated. Further, changes in uterine contractions preceding delivery are reported to be revealed by uEMG signals [2].

Several methods have been employed for characterizing the signals. Linear approaches assume stationary behavior of these signals and thus are unable to capture uEMG signal complexity. Since myocytes are intricately interconnected, they possess non-linear behavior. A comparative study stated non-linear methods to be efficient to analyze these signals [3]

Fractal analysis is a non-linear signal processing approach which evaluates the statistical measure of signal complexity. The signal fluctuations could be characterized using quantitative fractal measures. Hence, fractal analysis is a potential diagnostic tool for analyzing biosignals [4] 
Empirical Mode Decomposition based Detrended Fluctuation Analysis (EMD-DFA) is a fractal method that facilitates the investigation of physiological signals. In this technique, the signal non-stationarities are detrended using Empirical Mode Decomposition (EMD). It is reported to be more effective than conventional detrended fluctuation analysis [4].

In this study, an attempt has been made to comprehensively analyze preterm uEMG signals obtained during varied gestational ages using EMD-DFA.

\section{Methodology}

\section{1 uEMG Signals Database Description}

The uEMG signals are obtained from Term-Preterm Electrohysterogram database that is publicly available [3]. For this study, preterm signals that are acquired at different gestational weeks are considered.

The signals are obtained from three channels in bipolar configuration, using four electrodes placed on the abdomen surface. These electrodes are positioned symmetrically around the navel horizontally in two rows with a $7 \mathrm{~cm}$ distance between each other. The signals are sampled at $20 \mathrm{~Hz}$. For more details about the signal acquisition, the readers are referred to [3].

Due to artefact-free characteristics, third channel signals (recorded between two lowermost electrodes) are used for this work in the frequency range $0.3-3 \mathrm{~Hz}[3]$.

\subsection{Empirical Mode Decomposition based Detrended Fluctuation Analysis}

EMD-DFA is a fractal technique where the detrending is performed using the trends that are computed by EMD. The trend based on EMD is the residual that is obtained from the process of sifting. It is reported that the identification of trends by EMD improves the accuracy of trends [4].

The following steps are implemented in EMD-DFA. A signal $u(t)$ is divided into $N_{w}$ equal-sized non-overlapping segments. For each segment $u_{v}$, the EMD based local trend is obtained by the process of sifting.

EMD is applied to decompose the signal into intrinsic mode functions and residue $r_{n}(i)$. From each of the segments, this residue is detrended and local fluctuation $F(v, w)$ is calculated.

$$
F^{2}(v, w)=\frac{1}{w} \sum_{i=1}^{w}\left[u_{v}(i)-r_{n}(i)\right]^{2}, 1 \leq i \leq w
$$

Further, local fluctuation functions are averaged for all the segments and fluctuation function $F(w)$ is computed which has power-law relationship with scale $w$.

$$
F(w)=\sqrt{\frac{1}{N_{w}} \sum_{v=1}^{N_{w}} F^{2}(v, w)} \sim w^{H}
$$

\subsection{Fractal Feature Extraction and Statistical Analysis}

In a double-logarithmic plot, $\log _{2} F(w)$ is plotted in the ordinate axis against $\log _{2}(w)$ in abscissa. From this plot, distinct linear scaling regions are identified. Initially, to the whole set of abscissae, a linear regression line is fit for determining these scaling regions.

For the fit, Standard Error (SE) of slope and coefficient of determination $\left(R^{2}\right)$ are computed. This method is repeated by eliminating few values from the end of $\log _{2}(w)$ until $\mathrm{SE}<0.1$ and $R^{2}>0.95$ are achieved. The obtained region is referred to as the short-term scaling region. For the remaining values of $\log _{2}(w)$, a regression line is fit and the same approach is followed to obtain long-term scaling region [5].

Similarly, all the possible combinations of short- and long-term scaling regions are determined and the corresponding Chi-square $\left(\chi^{2}\right)$ statistic is computed for each combination. Further, according to $\chi^{2}$ test for independence, the combination of scaling regions with the minimum $\chi^{2}$ statistic is reported to be ideal and it is chosen for further analysis [6].

From the identified first and second scaling regions, slope-based and angle-based fractal features are extracted. Slope-based features are short-term exponent $(H s)$, long-term exponent $(H l)$ and Inflection Point (IP). The angle-based features include short-term $\left(\alpha_{H s}\right)$ and long-term $\left(\alpha_{H l}\right)$ fractal angles.

$H s$ is defined as slope of the linear fit of short-term scaling region. $\mathrm{Hl}$ refers to the slope of linear fit of long-term scaling region. $\mathrm{Hs}$ and $\mathrm{Hl}$ denotes the correlation properties of the signal for lower and higher values of scale $w$ respectively [5].

IP refers to intersection point in short-term and long-term regression lines. $\alpha_{H s}$ and $\alpha_{H l}$ are angles formed by short-term and long-term fits with the abscissa of double-logarithmic plots [7]. The Coefficient of Variation (CV) for the extracted features is calculated to examine the variations in different subjects. 


\section{Results and Discussion}

Two preterm signals recorded at different gestational weeks are shown in Figure 1. The time to delivery for the two signals is 11 weeks and 6.28 weeks of gestation respectively. For demonstrating the variations in the characteristics of preterm conditions during different gestational ages, these two representative signals have been randomly chosen.

It is observed that there is a variation in the signal amplitude which may be due to the difference in the rate of generation of action potentials and number of excited myocytes.

A higher amplitude is observed in the signals with lower time to delivery, as evident from Figure 1(b). This might be due to the rise in the uterine electrical activity as pregnancy progresses towards labor [2].

The double-logarithmic plot depicting the variation of $\log _{2}(F(w))$ with respect to $\log _{2}(w)$ for these preterm signals is shown in Figure 2. The value of $\log _{2}(F(w))$ in Figure 2(a) ranges from 1.22 to 4.46 . Similarly, $\log _{2}(F(w))$ varies between 2.08 and 5.29 in Figure 2(b).

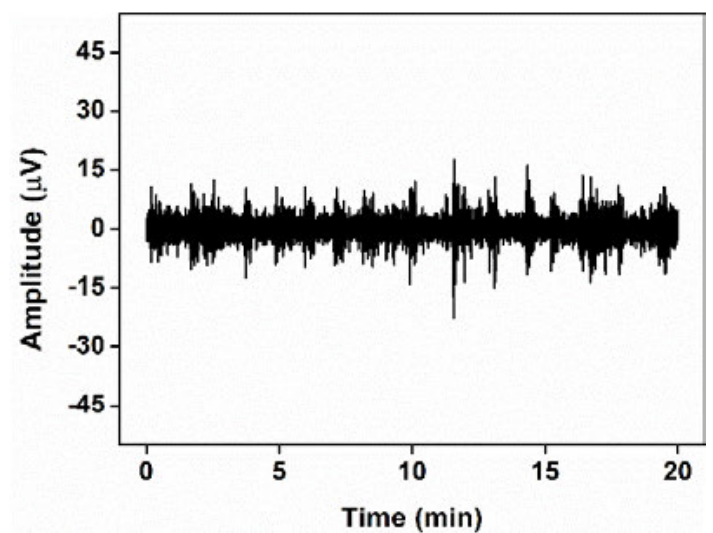

(a)

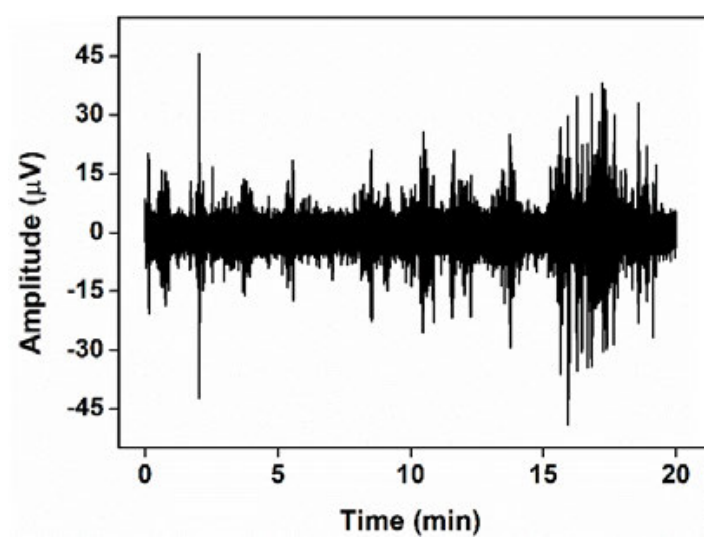

(b)

Figure 1: Representative preterm signals with time to delivery of (a) 11 weeks and (b) 6.28 weeks of gestation
The values of $\log _{2}(F(w))$ are found to vary with the gestational age. A slow variation of $F(w)$ is observed with $w$ when the time to delivery is more. This indicates the presence of rapid signal fluctuations when recorded in the early stages of pregnancy [5].

In addition, the short- and long-term scaling regions identified using $\chi^{2}$ method are illustrated in Figure 2. For the first signal, the $\chi^{2}$ statistic is 50.43, as shown in Figure 2(a). The $\chi^{2}$ statistic for the second signal is 61.59 , as depicted in Figure 2(b).

It is evident that there is an increase in slope-based feature values $H s$ and $H l$ as pregnancy progresses towards labor. This denotes the presence of strongly correlated signal fluctuations with increasing gestational weeks [5]. Further, mean and Standard Deviation (SD) of $H s$ and $H l$ features are $1.46(0.4)$ and $0.74(0.2)$ respectively.

The scatterplots depicting the variation of the angle-based features $\alpha_{H s}$ and $\alpha_{H l}$ with respect to the total gestational age are shown in Figure 3. $\alpha_{H s}$ feature varies between 42.11 and 68.86 while $\alpha_{H l}$ ranges from 23.02 to 49.77 . The average and SD values of $\alpha_{H s}$ and $\alpha_{H l}$ are 54.56 (6.7) and 36.04 (6.7) respectively.

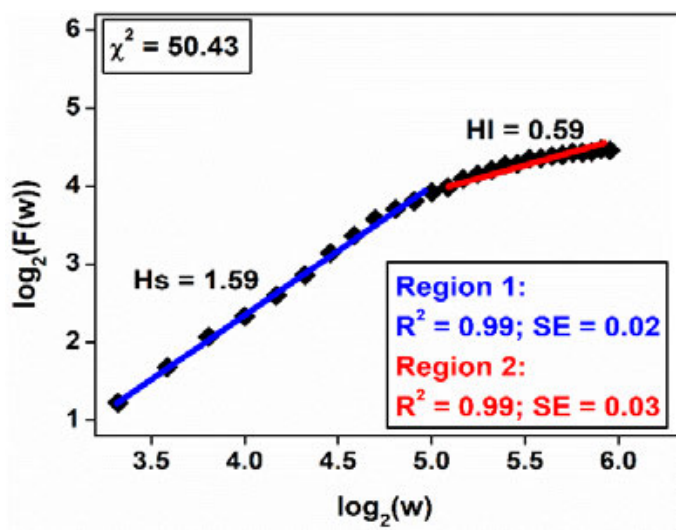

(a)

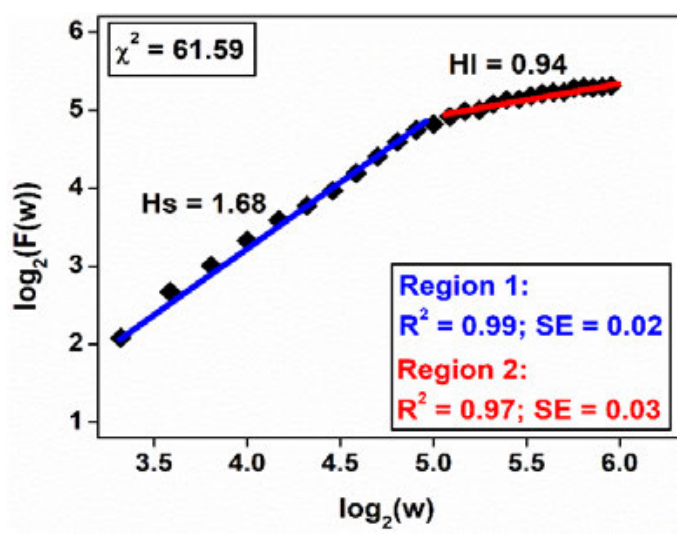

(b)

Figure 2: Logarithmic plots of $F(w)$ with separation of first and second scaling regions using $\chi^{2}$ method for representative preterm signals 


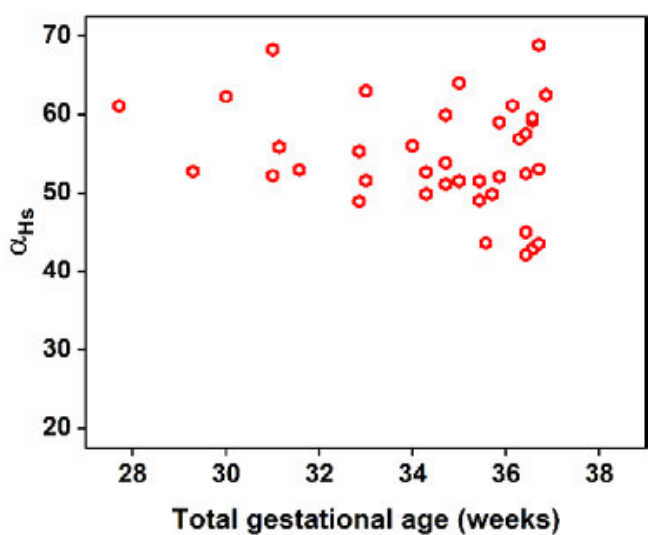

(a)

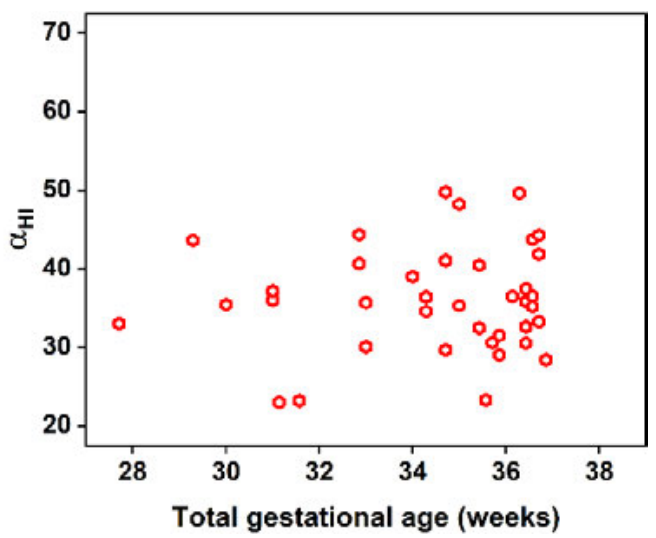

(b)

Figure 3: Scatterplots of angle-based features namely (a) $\alpha_{H s}$ and (b) $\alpha_{H /}$ with respect to total gestational age

Table 1: Variation of CV for fractal features extracted from preterm signals.

\begin{tabular}{cc}
\hline Features & CV \\
\hline$H s$ & 0.13 \\
$H I$ & 0.31 \\
$I P$ & 0.51 \\
$\alpha_{H s}$ & 0.11 \\
$\alpha_{H I}$ & 0.23 \\
\hline
\end{tabular}

The angle-based feature values indicate the presence of smoother signal fluctuations as pregnancy progresses towards labor [7]. This might be owing to the increased coordination of uterine contractions as the time to delivery approaches [5].

Table 1 denotes the variation of $\mathrm{CV}$ for the extracted fractal features. The least CV value is obtained for $\alpha_{H s}$ feature, while the highest CV is for IP feature. It is also seen that $H s$, $H l, \alpha_{H s}$ and $\alpha_{H l}$ have $\mathrm{CV}$ values less than 0.35 . This denotes that these features can depict the inter-subject variance. It is inferred that EMD-DFA features can comprehensively analyze preterm signal fluctuations.

\section{Conclusions}

In this work, preterm signals obtained at varied gestational ages are subjected to EMD-DFA. The signal fluctuations are distinct for varied weeks of preterm pregnancies. The fractal features are extracted to comprehensively analyze the signals. The feature values indicate presence of smoother fluctuations towards delivery. This might be due to synchrony in action potentials generated by the myocytes as time to delivery approaches.

It is demonstrated that EMD-DFA is able to analyze the subtle uEMG signal changes. As early preterm birth diagnosis is crucial for appropriate and effective medical intervention, the proposed approach appears to be useful in characterization of uterine contractions associated with preterm pregnancies.

\section{Author Statement}

Research funding: The authors state no funding involved.

Conflict of interest: The authors state no conflict of interest.

Informed consent: The authors have utilized the signals from an open-source database that followed the procedures.

Ethical approval: The data used in this study is considered from an open-source database that followed all these procedures.

\section{References}

[1] Euliano TY, Nguyen MT, Darmanjian S, McGorray SP, Euliano N, Onkala A, Gregg AR. Monitoring uterine activity during labor: a comparison of 3 methods. Am J Obstet Gynecol 2013; 208:66.e1-66.e6.

[2] Vlemminx MW, Thijssen KM, Bajlekov GI, Dieleman JP, Van Der Hout-Van MB, Jagt D, Oei SG. Could electrohysterography be the solution for external uterine monitoring in obese women. J Perinatol 2018; 38:580-586.

[3] Fele-Žorž G, Kavšek G, Novak-Antolic Ž, Jager F. A comparison of various linear and non-linear signal processing techniques to separate uterine EMG records of term and preterm delivery groups. Med Biol Eng Comput 2008; 46:911922.

[4] Yeh JR, Fan SZ, Shieh JS. Human heart beat analysis using a modified algorithm of detrended fluctuation analysis based on empirical mode decomposition. Med Eng Phys 2009; 31:92- 100.

[5] Punitha N, Vardhini P, Vinothini S, Ramakrishnan S. Analysis of fluctuations of uterine contractions in preterm pregnancies using adaptive fractal features of electromyography signals. Fluct Noise Lett 2021; 20:2150019.

[6] Weissman A, Binah O. The fractal nature of blood glucose fluctuations. J Diabetes Complications 2014; 28:646-651.

[7] Matić Z, Platiša MM, Kalauzi A, Bojić T. Slow $0.1 \mathrm{~Hz}$ breathing and body posture induced perturbations of RRI and respiratory signal complexity and cardiorespiratory coupling. Front Physiol 2020; 11:1-20. 